\title{
Wearable Pressure Sensing for Vojta Therapy Guidance
}

Steffen Büchner, Medical Informatics and Microsystems Engineering, University of Siegen, 57076 Siegen, Germany, steffen.buechner@uni-siegen.de

Christian Gibas, Medical Informatics and Microsystems, Engineering, University of Siegen, 57076 Siegen, Germany, Christian.gibas@uni-siegen.de

Nina Nemcová, Sozialpädiatrisches Zentrum der DRK Kinderklinik Siegen, International Vojta Society, 57076 Siegen, Germany, info@vojta.com

Wolfram Müller, Sozialpädiatrisches Zentrum der DRK Kinderklinik Siegen, International Vojta Society, 57076

Siegen, Germany, info@vojta.com

Katrin Schürholz, Sozialpädiatrisches Zentrum der DRK Kinderklinik Siegen, International Vojta Society, 57076 Siegen, Germany, info@vojta.com

Rainer Brück, Medical Informatics and Microsystems Engineering, University of Siegen, 57076 Siegen, Germany, rainer.brueck@uni-siegen.de

\section{Introduction}

The authors propose a glove with pressure sensitive fingertips and muscle tension detection of the thumb to aid in the physiotherapy of infants, children and adults according to Vojta. The therapy has to be carried out at home by the parents of children with cerebral palsy, or other movement disturbances up to 4 times per day. Often, parents struggle to carry out the practices because of insecurities regarding the therapy's proper application.

\section{Methods}

The presented glove is tailored towards the specific needs of Vojta therapy, e.g. flexibility and small sensor size, and can help parents gather objective data on their therapy application at home. It has been tested in multiple occasions on adult probands and seems to be a suitable tool to support parents and therapists in training alike.

\section{Results}

Our investigations allow for several scientific results. First, it has been shown that by means of a pressure sensitive glove a valuable support for parents and Vojta therapists can be implemented. A second benefit of our research is to objectify the Vojta-therapy itself. The approaches used in earlier work were not suitable for investigating what pressure was applied to the subjects. For adults, our measurements suggest that the pressure is relatively low and even decreases over the duration of the therapy. This is mostly the case if the intensity of the activation increases.

\section{Conclusion}

In this article the authors presented a pressure-sensitive glove specifically adapted to the needs of the Vojta therapy. We will also investigate to what extent such a device can be helpful in the training of future therapists that basically face the same uncertainty when starting their training. 


\section{In-silico adaptation of a myofibroblast electrophysiology model including intracellular calcium handling}

Jorge Sánchez, Institute of Biomedical Enginerring, Karlsruhe Institute of Technology (KIT), Karlsruhe, Germany, publications@ibt.kit.edu

Beatriz Trénor, Centro de Investigación e Innovación en Bioingeniería, Universitat Politècnica de València, Spain Javier Saiz, Centro de Investigación e Innovación en Bioingeniería, Universitat Politècnica de València, Spain Olaf Dössel, Institute of Biomedical Engineering, Karlsruhe Institute of Technology (KIT), Karlsruhe, Germany Axel Loewe, Institute of Biomedical Engineering, Karlsruhe Institute of Technology (KIT), Karlsruhe, Germany

\section{Introduction}

Recent studies have demonstrated that non-cardiomyocytes can be electrically connected to cardiomyocytes and could affect cardiac electric propagation. Myofibroblasts are cells responsible for maintaining the extracellular matrix, are electrically connected and can contract. However, the electrophysiology of myofibroblasts is still under study and especially the interaction of the intracellular calcium dynamics.

\section{Methods}

Single-cell in-silico experiments were set up and run using openCARP. The calcium current and the calcium intracellular handling was taken from Courtemanche et al. human atrial model and introduced to the myofibroblast model. Furthermore, using data from experimental studies measured in in-vitro pig/rats fibroblasts, a genetic algorithm was used to fit the calcium current curve minimizing the cost function of the root mean squared error. Lower and upper bounds were constrained to experimentally observed values $+/-10 \%$. The cell was left unpaced for 10 minutes and after paced at a basic cycle length of $1000 \mathrm{~ms}$ for 100 pulses to approach a stable limit cycle.

\section{Results}

Including the calcium ion current and intracellular handling from the human atrial model created an autonomic cyclic depolarization without pacing due to an activation of the calcium channel at the resting membrane potential of $-26 \mathrm{mV}$ of the myofibroblast without the calcium. After fitting using the genetic algorithm, the results of fitting the ionic currents, gave a more depolarized resting membrane potential compared to the cellular model without the calcium handling ( -44 $\mathrm{mV}$ and $-26 \mathrm{mV}$ respectively). The integral of the potassium currents were affected and the $\mathrm{IKv}_{\mathrm{v}}$ area was increased by $16 \%$ while the area of IK1 was reduced by $48 \%$.

\section{Conclusion}

Using genetic algorithm, myofibroblast electrophysiology model was fitted to reproduce experimental measurements. Calcium currents and intracellular handling changed the electrophysiology of the myofibroblast. Calcium mainly affects the flux of potassium through the cell membrane, which hyperpolarizes the resting membrane potential. 


\section{Automated incisional hernia characterization by non-rigid registration of CT images - a pilot study}

Samuel Voß, Forschungscampus STIMULATE; Department of Fluid Dynamics and Technical Flows, University of Magdeburg, Magdeburg, Germany, samuel.voss@ovgu.de

Philipp D. Lösel, Heidelberg Institute for Theoretical Studies, Heidelberg, Germany; Engineering Mathematics and Computing Lab, Interdisciplinary Center for Scientific Computing, Heidelberg, Germany, philipp.loesel@uniheidelberg.de

Sylvia Saalfeld, Forschungscampus STIMULATE; Department of Simulation and Graphics, University of Magdeburg, Magdeburg, Germany, sylvia@isg.cs.uni-magdeburg.de

Philipp Berg, Forschungscampus STIMULATE; Department of Fluid Dynamics and Technical Flows, University of Magdeburg, Magdeburg, Germany, berg@ovgu.de

Vincent Heuveline, Heidelberg Institute for Theoretical Studies, Heidelberg, Germany; Engineering Mathematics and Computing Lab, Interdisciplinary Center for Scientific Computing, Heidelberg, Germany, vincent.heuveline@uniheidelberg.de

Friedrich Kallinowski, General, Visceral and Transplatation Surgery, University Hospital Heidelberg, Heidelberg, Germany; General and Visceral Surgery, GRN Hospital Eberbach, Eberbach, Germany, friedrich.kallinowski@med.uni-heidelberg.de

\section{Introduction}

Incisional hernia repair makes use of prosthetic meshes to re-establish a biomechanically stable abdominal wall. Mesh sizing and fixation has been found to be essential for the clinical outcome. Comparative CT imaging a) under rest versus b) under Valsalva manoeuvre (exhalation against closed airways) provide useful information for hernia characterisation. However, this process incorporates several manual measurements which led to observer variability.

\section{Methods}

The present study suggests using an image registration approach of the CT data to extract hernia quantities reliably and reproducibly. The routine is implemented in Matlab and works fully automatic. After CT data import, layer by layer undergo non-rigid B-spline grid registration. Local displacement and strain are extracted from the transformation field.

\section{Results}

The qualitative results correspond to the clinical observation. Maximum displacement of $3.5 \mathrm{~cm}$ and maximum strain of $25 \%$ are calculated for one patient's data set.

\section{Conclusion}

Current approaches do not provide this type of information. Further research will focus on validation and possibilities to include this new kind of knowledge into the design process of prosthetic meshes. 


\section{Characteristics in Wideband Acoustic Immittance Measurements and their Underlying Mechanical Causes}

Benjamin Sackmann, Reutlingen Research Institute, Reutlingen University, 72762 Reutlingen, Germany, benjamin.sackmann@ reutlingen-university.de

Michael Lauxmann, Reutlingen Research Institute, Reutlingen University, 72762 Reutlingen, Germany, michael.lauxmann@reutlingen-university.de

\section{Introduction}

Wideband acoustic immittance (WAI) is a promising new audiological measurement method to measure the impedance of the ear with an acoustic stimulus testing several frequencies. However, the test results are not specific enough and therefore this measurement method is not accepted as standard in current clinical diagnosis. The aim of this study is to understand the relationship between pathology-specific characteristics in the WAI curves and their underlying mechanical causes in the middle ear.

\section{Methods}

For identifying the cause-effect relationship of features in WAI measurements and their corresponding mechanical causes a finite-element model of the ear is used. The model is adapted to a representative experiment described in literature in which different middle-ear pathologies were investigated in a single temporal bone and in addition to audiological measurements also ossicles motion were measured.

\section{Results}

The spatial distribution of stiffness along the ossicular chain is crucial to be able to match each of the pathologies equally well using a single model. Different types of measurement data is essential for the parameter identification, otherwise the parameter choice is ambiguous. Disarticulation is characterized by a resonance peak between 0.6 and $0.9 \mathrm{kHz}$, mainly caused by a reduction in the stiffness of the incudostapedial joint and decoupling of the cochlea damping. Malleus fixation and otosclerosis are characterized by a resonance shift to higher freqencies with an increase in impedance mainly below $1 \mathrm{kHz}$ which is caused by an increase in translational and rotational stiffness of the superior malleolar ligament and in case of otosclerosis by an increase of stiffness of the annular ligament.

\section{Conclusion}

Ambiguities in model parameters, lead to the large parameter variations described in literature. While disarticulation is clearly distinguishable from the investigated pathologies, otosclerosis and malleus fixation differ slightly in the frequency range between 1 and $2 \mathrm{kHz}$ by a lower impedance for otosclerosis. 
Ekaterina Kovacheva ${ }^{\dagger *}$, Lukas Baron ${ }^{\dagger}$, Steffen Schuler, Tobias Gerach, Olaf Dössel, and Axel Loewe

\section{Optimization Framework to Identify Constitutive Law Parameters of the Human Heart}

Over the last decades, computational models have been applied in in-silico simulations of the heart biomechanics. These models depend on input parameters. In particular, four parameters are needed for the constitutive law of Guccione et al., a model describing the stress-strain relation of the heart tissue. In the literature, we could find a wide range of values for these parameters.

In this work, we propose an optimization framework which identifies the parameters of a constitutive law. This framework is based on experimental measurements conducted by Klotz et al.. They provide an end-diastolic pressure-volume relation- ship. We applied the proposed framework on one heart model and identified the following elastic parameters to optimally match the Klotz curve: $C=313 \mathrm{~Pa}, b_{f}=17.8, b_{t}=7.1$ and $b_{f t}=12.4$.

In general, this approach allows to identify optimized parameters for a constitutive law, for a patient-specific heart geometry. The use of optimized parameters will lead to physiological simulation results of the heart biomechanics and is therefore an important step towards applying computational models in clinical practice.

${ }^{*}$ Corresponding author: Ekaterina Kovacheva ${ }^{\dagger}$, Institute of Biomedical Engineering, Karlsruhe Institute of Technology (KIT), Kaiserstr. 12, 76131 Karlsruhe, Germany, e-mail: publications@ibt.kit.edu

Lukas Baron ${ }^{\dagger}$, Steffen Schuler, Tobias Gerach, Olaf Dössel, Axel Loewe, Institute of Biomedical Engineering, Karlsruhe In- stitute of Technology (KIT), 76131 Karlsruhe, Germany. ${ }^{\dagger}$ These authors contributed equally. 